\title{
COMMUTATIVE, NOETHERIAN RINGS OVER WHICH EVERY MODULE HAS A MAXIMAL SUBMODULE
}

\author{
ROSS M. HAMSHER ${ }^{1}$
}

In $[1$, p. 470] Professor Hyman Bass mentions the following conjecture: a ring $R$ is left perfect if, and only if, every nonzero left $R$-module has a maximal submodule and $R$ has no infinite set of orthogonal idempotents. If a ring $R$ is right or left noetherian, then $R$ has no infinite set of orthogonal idempotents. We shall show that for commutative, noetherian rings Bass' conjecture is true.

LEммA. If $R$ is a commutative ring over which every nonzero module has a maximal submodule, then every proper prime ideal of $R$ is maximal.

Proof. Let $P$ be a proper prime ideal of $R$ so $S=R / P$ is an integral domain over which every nonzero module has a maximal submodule. Let ${ }_{s} Q$ be a nonzero, injective $S$-module. Then ${ }_{s} Q$ has a simple epimorphic image, say $S / M$ where $M$ is a maximal ideal of $S$. If $m \in M$ and $m \neq 0$, then, being the quotient of an injective module, $S / M$ is divisible, and there is an $s \in S$ with $m(s+M)=m s+M=1+M$. Hence $1 \in M$, a contradiction. Thus $M=0$, and $S$ is a field.

If ${ }_{R} M$ is an $R$-module, then ${ }_{R} M$ is $R$-projective if for each epimorphism $\sigma:{ }_{R} R \rightarrow{ }_{R} C$ and each homomorphism $\pi:{ }_{R} M \rightarrow{ }_{R} C$, there is a homomorphism $\tau:{ }_{R} M \rightarrow{ }_{R} R$ such that $\tau \sigma=\pi$. We call a ring $R$ a test module for projectivity if every $R$-projective module is projective.

THEOREM 1. Let $R$ be a commutative, noetherian ring. Then the following are equivalent.

(i) $R$ is a test module for projectivity,

(ii) every nonzero $R$-module has a maximal submodule, and

(iii) $R$ is artinian.

Proof. (iii) $\Rightarrow(\mathrm{i})$. As Professor Barbara L. Osofsky shows in [3], this implication follows from Sandomierski [4, Theorems 4.1 and 4.4].

(i) $\Rightarrow$ (ii): Every nonzero projective module has a nonzero homo-

Received by the editors June 6, 1966.

1 This note forms a part of the author's Henry Rutgers thesis written at Rutgers, the State University, under the direction of Professor Barbara L. Osofsky on a project sponsored by the National Science Foundation through Undergraduate Research Participation Grants GE-1886, GE-4206 and GE-6287. 
morphic image in a cyclic. If a module $M$ has no nonzero homomorphic image in a cyclic, $M$ is trivially $R$-projective so by (i) $M$ is projective and $M=0$. Thus, if $M$ is a nonzero module, there exists a nonzero homomorphism $\sigma$ of $M$ to a cyclic. Then $\operatorname{Im} \sigma$ is finitely generated, so Im $\sigma$ has a maximal submodule, and so must $M$.

(ii) $\Rightarrow$ (iii). It suffices to show that $R$ is perfect by a remark in Bass [1, p. 475]. Since $R$ has no infinite set of orthogonal idempotents, $R$ is perfect if every nonzero $R$-module has a simple submodule [1]. Let $M$ be a nonzero $R$-module with $m \in M, m \neq 0$. Select a maximal ideal from $\{(0: r m): r \in R, r m \neq 0\}$, say $(0: s m)$. Suppose that $a b$ $\in(0: s m)$, but $a \notin(0: s m)$. Then $a s m \neq 0$ and $(0: s m) \subset(0: a s m)$ imply $(0: s m)=(0: a s m)$. This shows that $b \in(0: s m)$, and $(0: s m)$ is a proper prime ideal of $R$. By the lemma $(0: s m)$ is maximal so $R s m$ is simple.

TheOREM 2. A commutative ring $R$ is perfect if, and only if, every nonzero $R$-module has a maximal submodule and $R / J$ (where $J$ is the Jacobson radical of $R$ ) satisfies the ascending chain condition on the annihilators of principal ideals.

Proof. Clearly if $R$ is perfect the second part of the theorem holds; see [1]. Conversely, if the second part of the theorem holds, then by a remark in $[1$, p. 470] to show $R$ is perfect it suffices to show that $R / J$ is semi-simple artin. The condition on annihilator ideals implies that $R / J$ has no infinite set of orthogonal idempotents. Then using the obvious modification of the technique in (ii) $\Rightarrow$ (iii) above, we obtain that $R / J$ is an essential extension of its socle. Thus $R / J$ is semi-simple artin.

\section{REFERENCES}

1. H. Bass, Finitistic dimension and a homological generalization of semi-primary rings, Trans. Amer. Math. Soc. 95 (1960), 466-488.

2. B. Eckmann and A. Shopf, Über injective moduln, Arch. Math. 4 (1953), 75-78.

3. B. Osofsky, Global dimension af valuation rings (to appear).

4. F. Sandomierski, Relative injectivity and projectivity, Ph.D. Thesis, Penn. State Univ., University Park, Penn., 1964.

Rutgers, The State University 